\title{
Early Patient Access to Medicines: Health Technology Assessment Bodies Need to Catch Up with New Marketing Authorization Methods
}

\author{
Lada Leyens Angela Brand \\ Maastricht Economic and Social Research Institute on Innovation and Technology (MERIT), Maastricht University, \\ Maastricht, The Netherlands
}

\begin{abstract}
Key Words
Early dialogue $\cdot$ Marketing authorization application . Health technology assessment · European Medicines Agency · Food and Drug Administration - Adaptive pathways $\cdot$ Innovative medicines
\end{abstract}

\begin{abstract}
National and international medicines agencies have developed innovative methods to expedite promising new medicines to the market and facilitate early patient access. Some of these approval pathways are the conditional approval and the adaptive pathways by the European Medicines Agency (EMA); the Promising Innovative Medicine (PIM) designation and the Early Access to Medicines Scheme (EAMS) by the Medicines and Healthcare Products Regulatory Agency (MHRA), as well as the Fast Track, Breakthrough or Accelerated Approval methods by the Food and Drug Administration (FDA). However, at least in Europe, these methods cannot achieve the goal of improving timely access for patients to new medicines on their own; the reimbursement process also has to become adaptive and flexible. In the past 2 years, the effective access (national patient access) to newly approved oncology drugs ranged from 1 to 30 months, with an extremely high variability be-
\end{abstract}

tween European countries. The goal of early patient access in Europe can only be achieved if the national health technology assessment bodies, such as NICE (ENG), HAS (FR), G-BA (DE) or AIFA (IT), provide harmonized, transparent, flexible, conditional and adaptive methods that adopt the level of evidence accepted by the medicines agencies. The efforts from medicines agencies are welcome but will be in vain if health technology assessments do not follow with similar initiatives, and the European 'postcode' lottery will continue.

(C) 2016 The Author(s) Published by S. Karger AG, Basel

\section{New Approaches to Marketing Authorizations of Medicines}

\section{Status Quo}

Until recently, the development of medicines had a very rigid and fixed structure: safety and the first proofs of concept were tested in preclinical trials; if the results were promising, the first tests in humans started with phase I clinical trials, followed by clinical trial phases II and III that tested the medicine's efficacy and safety in the appropriate patient population. We used to talk about a 10 -year development process, costing around USD 1 bil-

\section{KARGER \\ E-Mail karger@karger.com} www.karger.com/phg

\section{The Author(s) \\ Published by S. Karger AG, Basel 1662-4246/16/0193-0187\$39.50/0}

This article is licensed under the Creative Commons AttributionNonCommercial-NoDerivatives 4.0 International License (CC BY NC-ND) (http://www.karger.com/Services/OpenAccessLicense) Usage and distribution for commercial purposes as well as any distribution of modified material requires written permission. 


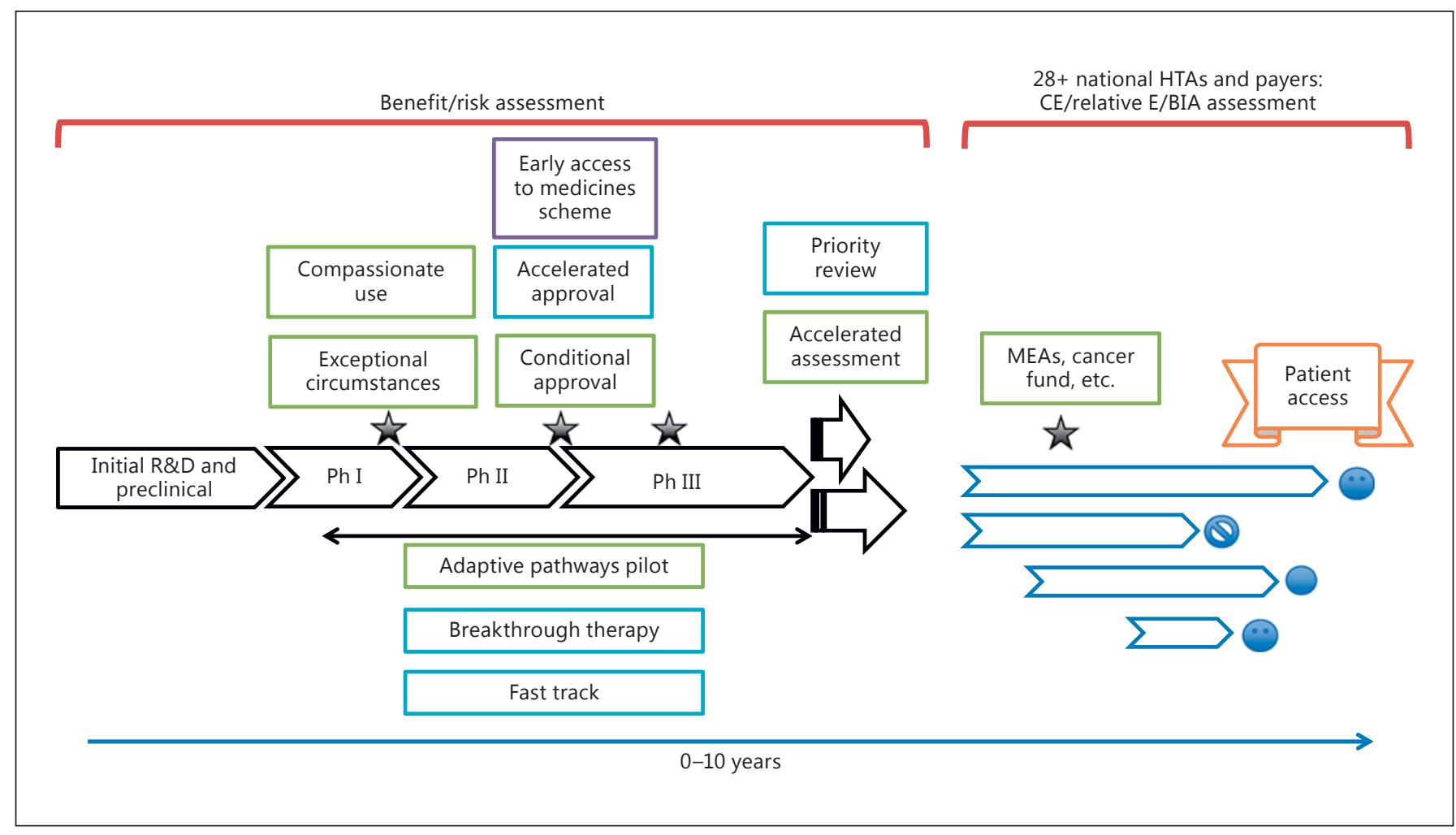

Fig. 1. Available regulatory tools for early dialogue and early patient access placed on the current drug development phases from initial R\&D to the market. Green and purple boxes are for available programs in the EU and blue boxes are for available programs in the USA. Modified from [3]. BIA = Budget impact analysis; $\mathrm{CE}=$ cost effectiveness; MEAs = managed entry agreements; $\mathrm{Ph}=$ clinical trial phase; $\mathrm{R} \& \mathrm{D}=$ research and development; Relative $\mathrm{E}=$ relative effectiveness. lion where only $1 \%$ of medicines initially tested made it to the market [1]. Thanks to the latest developments in science and technology, we understand diseases better and are able to design more targeted drugs, shortening the development process and avoiding the massive fallout of drug candidates on the way to the market. Moreover, new simulation mechanisms and clinical trial designs are used to expedite the development even further [2].

Marketing authorization pathways also used to follow a rigid and fixed structure. Only in exceptional circumstances could a drug be approved before completing phase III clinical trials with large number of patients (hundreds to thousands). The available conditional approvals were not used often and mainly for medicines that did not make it through the normal approvals pathway [3]. Some countries implemented methods to allow very early patient access to unapproved medicines, if physicians thought they could be beneficial for individual patients; an example is the German 'Heilversuch' (named patient access) approved for each individual patient by the national medicines agency [4].

\section{New Approaches}

The European Medicines Agency (EMA), Food and Drug Administration (FDA), Medicines and Healthcare Products Regulatory Agency (MHRA) and other medicines agencies have recently developed innovative marketing authorization pathways to facilitate early patient access to innovative and promising new medicines, in order to shorten the long time some patients have to wait until they are able to access new effective medicines (fig. 1). These pathways are either fully implemented, such as the Fast Track, Breakthrough or Accelerated Approval methods by the FDA, and the Early Access to Medicines Scheme (EAMS) by the MHRA, or in a pilot phase, such as the 'Medicines Adaptive Pathways to Patients' (MAPPs) by the EMA [3]. These new approaches are very well defined in each regulator's website and will not be further described in this article. 


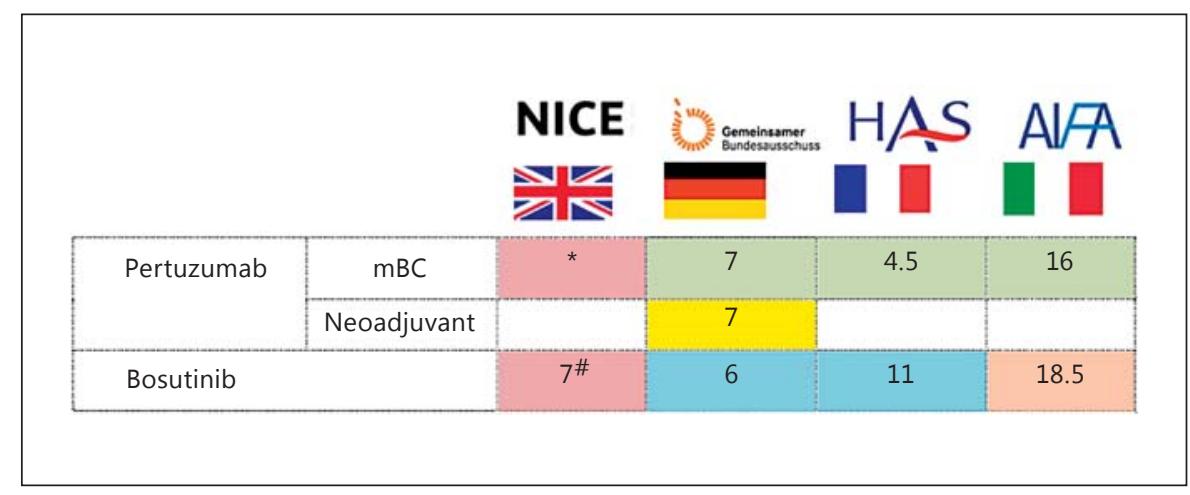

Fig. 2. Pricing and reimbursement decisions for pertuzumab (both indications have been separated) and bosutinib in England, Germany, France and Italy. Numbers refer to the delay in decision by national bodies after EC approval (in months). Red = Not recommended; green = recommended with added value; yellow $=$ recommended, no added value; blue = recommended, added value not quantifiable; orange $=$ reimbursed no information on added value;

\section{Common Denominators}

These pathways encourage an early and continuous dialogue between all stakeholders, including manufacturers, applicants, regulators, patients and physicians. For example, the adaptive pathways pilot launched by the EMA in 2014 promotes early discussions between the developer and the agency, as well as early involvement of patients and health technology assessment (HTA) bodies. Real-life evidence that supplements clinical trial data is also a common denominator in many of these pathways. Regulators are starting to accept to review real-life data in innovative marketing authorization application (MAA) methods. The iterative development of medicine is another new common factor; it can be achieved by either approving the medicine in stages with restricted populations at first, or by using conditional approvals and surrogate endpoints that have to be confirmed with more mature data later on [5].

A good example of multi-stakeholder dialogue is the EMA-HTA Scientific Advice procedure. Regulators, manufacturers, HTA bodies, patients, physicians and other experts discuss the questions raised by the manufacturer in one meeting. The patient's and expert's points of view are taken into account and considered by all other parties. At the national level, the EAMS also promotes early multi-stakeholder dialogue between the manufacturer, MHRA, NICE and the NHS [6].

HTA Bodies and New MAA Methods white $=$ no decision yet $; \mathrm{mBC}=$ metastatic breast cancer; ${ }^{*}=$ no final decision has been reached by NICE; the preliminary decision from August 6, 2013 does not recommend pertuzumab; a special committee is studying this and similar cases; ${ }^{\#}=$ bosutinib was in a rapid reconsideration process at the time of article publication with decision publication expected by October 2016. Modified from [5].

\section{Differences in Approval Times and Need for New Flexible HTA Methods}

In order to illustrate the difference in effective patient access to medicines in Europe, we will present the case of two cancer drugs: pertuzumab (Perjeta ${ }^{\mathrm{TM}}$ ) and bosutinib (Bosulif $\left.{ }^{\mathrm{TM}}\right)$. The delay in the publication of an HTA/reimbursement decision after EC approval, which directly affects patient access, could be up to 18 months in these examples. Furthermore, each national HTA body imposed different restrictions and even had completely different conclusions on the acceptability of the drug for their national patients. The paper by Martinalbo et al. [7] discusses in great detail the differences in pricing and reimbursement decisions and approval times at the national level.

Pertuzumab was approved in March 2013 for patients with metastatic breast cancer and in July 2015 for the neoadjuvant treatment of patients with breast cancer. The orphan drug bosutinib was conditionally approved in March 2013 for patients with Philadelphia chromosomepositive chronic myelogenous leukemia. As we can see in figure 2, AIFA was the body with longer decision delays in these examples. In the case of pertuzumab, NICE ruled a preliminary decision of not recommending the drug, in contrast to G-BA who recommends its use and suggests that it provides considerable added value in one of its indications. For bosutinib, NICE issued a negative opinion and it is re-examining the evidence within a rapid re-con- 
sideration process due to end in October 2016. All other bodies recommended bosutinib even if they agreed that the added value could not be quantified based on the limited information available at the time of MAA.

We have to consider the vast differences in the methods used by these bodies to evaluate medicines. The biggest difference between the bodies evaluated in this paper is the fact that NICE uses cost-effectiveness analysis for its evaluations, whereas G-BA, HAS and AIFA perform a relative effectiveness analysis. This can be one of the reasons for the disparities in final decisions. Whatever the methods are, the truth is that these differences directly affect patient access in Europe, in these cases to the detriment of English patients.

\section{New Proposals for HTAs and Conclusion}

As we have seen in figure 2, the pricing and reimbursement landscape in Europe is too heterogeneous. There are many other examples, and an analysis of many approved drugs would give the same picture. This directly affects patient access. Unfortunately, the efforts from the EMA and other regulatory agencies to facilitate early patient access to innovative medicines are 'diluted' in the pricing and reimbursement ( $\mathrm{P} \& \mathrm{R})$ and HTA process by long delays.

Even though they constitute big steps forward, the 'early dialogue' and the 'multi-stakeholder dialogue' promoted by the MAPPs pilot and the EMA-HTA scientific advice procedure are voluntary procedures and are not implemented in every medicine's development. The ideal future situation would entail the inclusion of systematic early and multi-stakeholder dialogue in all medicine developments that have reached certain milestones and prove to be promising.

It is clear that patients' safety should be at the forefront of all stakeholders' intentions. As some critics of flexible approval methods are expressing their concerns on these methods and arguing that they endanger patients' safety, it is important to remind them that regulatory agencies know their mandates and discuss in great detail all decisions, always having the patients' best interest in mind. We have to be able to find the right balance between acceptable early evidence for determining a positive risk/benefit balance and the risks associated with limited information. If the new flexible approval pathways allow restricted and controlled patient access, this risk can be well controlled and immediate actions can be taken when safety signals appear.

To avoid the delay in patient access and the European 'post-code' lottery, we need a more coordinated HTA process at the European level. As the ENVI report [7] proposes, relative effectiveness could be evaluated centrally with a common conclusion that is then applied nationally or locally by each country or region according to its applicable priorities and regulations. Another important aspect is to link the HTA and the MAA processes. They evaluate very similar information on individual products; it is therefore a waste of resources that all HTA/reimbursement bodies re-analyze the same information the medicine agencies have already studied in detail. In this direction, the EMA is considering the possibility to include more HTA-relevant information in their CHMP assessment report in order to facilitate the work of their HTA colleagues and the reimbursement evaluation process [8]. But most importantly, HTA and reimbursement bodies have to leave behind the rigid evaluation methods and adopt the flexible methods that are being implemented by medicine agencies. This is the only way to offer patients an effective early access to medicines. Nevertheless, we have to take into account that HTA bodies are 'younger' bodies than medicine agencies; it is therefore possible that they need more time to reach the 'maturity' and experience that medicine agencies needed in order to start implementing flexible approaches that force them out of the comfort zone of nearly total assurance in their risk/ benefit decisions.

As essential next steps, we can consider the need to provide more insights into improving HTA techniques on the level of research. Furthermore, we need to extend the reach of HTA to all other aspects of health, beyond medicines and medical devices. On the European political level, we need a unified approach for the determination of relative effectiveness for medicines and medical devices, and most importantly, on the policy level, we need new flexible approaches in order to catch up with the ones from medicine agencies to facilitate early patient access. The important goal of early patient access to promising medicines in Europe can only be achieved if the national health technology assessment bodies, such as NICE (ENG), HAS (FR), G-BA (DE) or AIFA (IT), provide harmonized, transparent, flexible, conditional and adaptive methods that adopt the level of evidence accepted by the medicine agencies. The efforts from medicines agencies are essential but will be in vain if HTAs do not follow with similar initiatives, and the European 'post-code' lottery will continue.

\section{Disclosure Statement}

The authors declare no conflicts of interest.
Leyens/Brand 


\section{References}

1 Adams C, Bran V: Spending on new drug development. Health Economics 2010;19:130141.

2 Berntgen M, Gourvil A, Pavlovic M, Goettsch W, Eichler H-G, Kristensen F: Improving the contribution of regulatory assessment reports to health technology assessments - a collaboration between the European Medicines Agency and the European network for Health Technology Assessment. Value Health 2014; 17:634-641.

3 European Parliament: Towards a Harmonised EU Assessment of the Added Therapeutic Value of Medicines. Policy Department A:
Economic and Scientific Policy. Brussels, European Union, 2015.

4 Hart D: Heilversuch und klinische Prüfung. Kongruenz und Differenz. Medizinrecht 2015;33:766-775.

5 Leyens L, Richer É, Melien $\emptyset$, Ballensiefen W, Brand A: Available tools to facilitate early patient access to medicines in the EU and the USA: analysis of conditional approvals and the implications for personalized medicine. Public Health Genomics 2015;18:249_ 259.

6 Lockwood P, Ewy W, Hermann D, Holford N: Application of clinical trial simulation to compare proof-of-concept study designs for drugs with a slow onset of effect; an example in Alzheimer's disease. Pharm Res 2006;23: 2050-2059.

$>7$ Martinalbo J, Bowen D, Camarero J, Chapelin M, Démolis P, Foggi P, et al: Early market access of cancer drugs in the EU. Ann Oncol 2016;27:96-105.

8 NICE: Note to describe procedures at NICE to support the early access to medicines scheme. https://www.nice.org.uk/Media/Default/About/Who-we-are/Policies-and-procedures/eams-process-jan-16.pdf (accessed April 10, 2016). 